Part of Journal of Research of the National Bureau of Standards, Volume 22, May 1939

\title{
TORQUE BETWEEN CONCENTRIC SINGLE-LAYER COILS
}

\author{
By Chester Snow
}

\section{ABSTRACT}

This paper is concerned with several types of small correction terms which must be taken into account when computing the torque or the mutual inductance between two concentric single-layer helices. These are to be added to the formula previously developed for two current sheets. The seven types of corrections are those which may be foreseen from experience with other absolute electrical measurements. The corrections (a) to $(\mathrm{g})$ are given in section $\mathrm{I}$.

\section{CONTENTS}

I. Introduction 1 Page

II. Mutual inductance between two circular filaments, $f_{1}$ and $f_{2}$, whose axes intersect at an angle, $\theta \ldots \ldots$.

III. Mutual inductance between a sheet and a filament........... 610

IV. Mutual inductance between two turns of wire . . . . . . . . . . 611

V. Mutual inductance of the coils

VI. Torque including the first four corrections _...

VII. Effect of errors in centering the coils _._.

VIII. Effect of axial components of current

IX. Effect of lead-in wires _...

X. Summary _............

\section{INTRODUCTION}

The mutual inductance, $M$, from which the torque is derived by differentiation with respect to the angle, $\theta$, between their axis, consists principally of that between two equivalent current sheets. A formula for the latter is derived in Bureau Research Paper RP24, eq 32. ${ }^{1}$ In applying it to the case of actual single-layer coils, formula 35 , a factor $\cos \alpha_{2}$ was written in front of the series, which should have been $1 / \cos$ $\alpha_{2}$. With this correction, the formula may be put in the following form, where $N_{1}$ and $N_{2}$ are the total number of turns, $l_{1}$ and $l_{2}$ the lengths, $a_{1}$ and $a_{2}$ the radii, $r_{1}$ and $r_{2}$ the semidiagonals, and $\mu_{1}$ and $\mu_{2}$ are $\cos \alpha_{1}, \cos \alpha_{2}$, where $\alpha_{1}$ and $\alpha_{2}$ are the angles between the diagonals of the coils and their respective axes and $\mu=\cos \theta$.

$$
\begin{aligned}
M_{s 1 s 2}\left(l_{1}, a_{1} ; l_{2} a_{2} ; \mu\right)= & 4 \pi \frac{N_{1}}{l_{1}} \pi a_{2}^{2} N_{2} \cdot \mu_{1} . \\
& \cdot\left\{\mu-\left(\frac{1-\mu_{1}^{2}}{2}\right) \sum_{n=1}^{\infty}\left(\frac{r_{2}}{r_{1}}\right)^{2 n} \cdot C_{n}\left(\mu_{1}\right) C_{n+1}\left(\mu_{2}\right) P_{2 n+1}(\mu)\right\},
\end{aligned}
$$

${ }_{1}$ Chester Snow, Mutual inductance and torque between two concentric solenoids, BS J. Research 1, 685 (1928) RP24, 
where

$C_{n}(\mu)=\frac{2 P^{\prime}{ }_{2 n}(\mu)}{2 n(2 n+1) \mu}=F\left(1-n, n+\frac{3}{2}, 2 ; 1-\mu^{2}\right)$

and

$$
=\frac{-2(-1)^{n} \Gamma\left(n+\frac{1}{2}\right)}{\sqrt{\pi}} \frac{}{\Gamma(n+1)} F\left(1-n, n+\frac{3}{2}, \frac{3}{2} ; \mu^{2}\right)
$$

$P_{n}(\mu)$ is Legendre's polynomial and $P_{n}^{\prime}(\mu)$ is $\frac{d P_{n}(\mu)}{d \mu}$. The subscript 1 refers to the outer current sheet, $s_{1}$, and subscript 2 to the inner sheet, $s_{2}$.

The small corrections to be found are:

(a) That which replaces the current sheet by a system of parallel, equally spaced turns of wire (instead of the tape windings with no

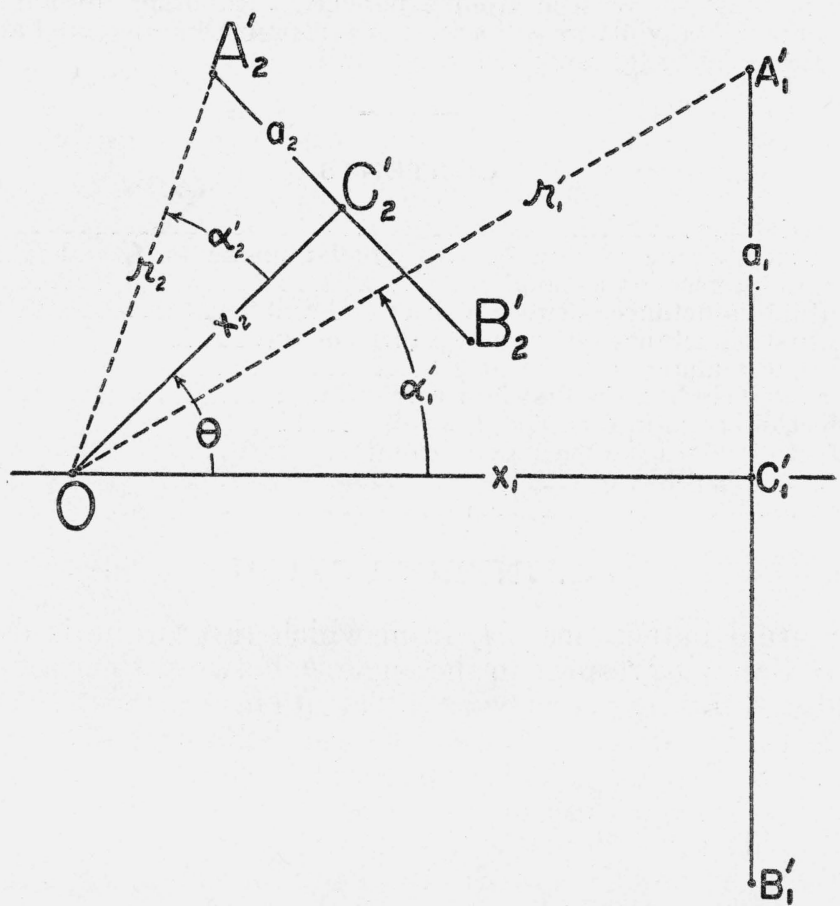

Frgure 1.-Section containing the intersecting axes of two circular filaments $A_{1}^{\prime} C_{1}^{\prime} B_{1}^{*}$ and $A_{2}^{\prime} C_{2}^{\prime} B_{2}^{\prime}$.

radial thickness or insulating space between them which constitute a current sheet).

(b) The effect of the nature of the current distribution over the sections of the wire. Two cases are considered: Uniform distribution and the "natural" one which is inversely proportional to the distance from the axis of the solenoid.

(c) The effect of small variations, $u_{a_{1}}\left(x_{1}\right)$ [and $u_{a_{2}}\left(x_{2}\right)$ ], in the radii of the turns, which are observed functions of the distance, $x_{1}$ (and $x_{2}$ ), respectively, of the turn from the origin at the center of the coils. 
(d) The effect of small variations in pitch, that is, of the small observed axial displacement, $u_{x}\left(x_{1}\right)$, (and $u_{x}\left(x_{2}\right)$ ) of the turn from the position corresponding to uniform spacing.

(e) The effect of errors in centering the coils.

(f) That due to the fact that the coils are helical and not a system of parallel turns of wire. This is the effect of the axial component of current.

(g) The contribution of lead-in wires.

\section{MUTUAL INDUCTANCE BETWEEN TWO CIRCULAR FILAMENTS, $f_{1}$ AND $f_{2}$, WHOSE AXES INTERSECT AT AN ANGLE, $\theta$}

A section by a plane containing the intersecting axes is shown in figure 1. The trace of $f_{1}$ is $A_{1}^{\prime} C_{1}^{\prime} B_{1}^{\prime}$ of $f_{2}$ is $A_{2}^{\prime} C_{2}^{\prime} B_{2}^{\prime}$. Let

$$
\begin{aligned}
& \overline{O A}_{1}^{\prime}=r_{1}^{\prime} ; \overline{O C}_{1}^{\prime}=x_{1}=r_{1}^{\prime} \cos \alpha_{1}^{\prime} ;{\overline{C_{1}^{\prime}}}_{1}^{\prime}=a_{1}=r_{1}^{\prime} \sin \alpha_{1}^{\prime} \\
& \overline{O A}_{2}^{\prime}=r_{2}^{\prime} ; \overline{O C}_{2}^{\prime}=x_{2}=r_{2}^{\prime} \cos \alpha_{2}^{\prime} ;{\overline{C_{2}^{\prime}}}_{2}^{\prime}=a_{2}=r_{2}^{\prime} \sin \alpha_{2}^{\prime} \\
& \mu_{1}^{\prime}=\cos \alpha_{1}^{\prime} ; \mu_{2}^{\prime}=\cos \alpha_{2}^{\prime} \mu=\cos \theta .
\end{aligned}
$$

If filament $f_{1}$ is any section of the larger current sheet, $s_{1}$, and filament $f_{2}$ of the inner sheet, $s_{2}$, which lies entirely within $s_{1}$ for any value of $\theta$, then $r_{2}^{\prime}<r_{1}^{\prime}$. In this case the following series ${ }^{2}$ has been shown to converge and to represent the mutual inductance, $M_{f_{1} f_{2}}$, of the two filaments when the angles $\alpha_{1}^{\prime}, \alpha_{2}^{\prime}$, and $\theta$ each lie in the range from zero to $\pi$, inclusive.

$$
\begin{aligned}
& M_{f_{1} \gamma_{2}}\left(x_{1}, a_{1} ; x_{2}, a_{2} ; \mu\right) \\
& =4 \pi^{2} \sum_{n=1}^{\infty}\left[\frac{\left(1-\mu_{1}^{\prime 2}\right) P_{n}^{\prime}\left(\mu_{1}^{\prime}\right)}{\left(r_{1}^{\prime}\right)^{n}}\right]\left[\left(r_{2}^{\prime}\right)^{n+1}\left(1-\mu_{2}^{\prime 2}\right) P_{n}^{\prime}\left(\mu_{2}^{\prime}\right)\right] \frac{P_{n}(\mu)}{n(n+1)}
\end{aligned}
$$

It is readily found that the functions $V=\left(1-\mu^{2}\right) r^{-n} P_{n}^{\prime}(\mu)$ and $V=r^{n+1}$ $\left(1-\mu^{2}\right) P_{n}^{\prime}(\mu)$ are solutions of the partial differential equation

that is,

$$
\left(D_{r}^{2}+\frac{1-\mu^{2}}{r^{2}} D_{\mu}^{2}\right) V=0
$$

$$
\left(D_{x}^{2}+D_{a}^{2}-\frac{1}{a} D_{a}\right) V=0 .
$$

Hence, $M_{f_{1} f_{2}}$ satisfies the equation

$$
\left(D_{x_{1}}^{2}+D_{a_{1}}^{2}-\frac{1}{a_{1}} D_{a_{1}}\right) M_{f_{1} f_{2}}=0
$$

in the variables $x_{1}$ and $a_{1}$, and the similar equation

$$
\left(D_{x_{2}}^{2}+D_{a_{2}}^{2}-\frac{1}{a_{2}} D_{a_{2}}\right) M_{f_{1} f_{2}}=0
$$

in the variables $x_{2}$ and $a_{2}$, which are placed here for later reference.

${ }^{2}$ Chester Snow, Mutual inductance of any two circles, BS J. Research 1, 531 (1928) RP18. (See eq 23, p. 539.) 
610 Journal of Research of the National Bureau of Standards [Vol.22

\section{MUTUAL INDUCTANCE BETWEEN A SHEET AND A FILAMENT}

The mutual inductance of the two current sheets, $M_{s_{1} s_{2}}$, given in eq 1 was found by multiplying $M_{f_{1} f_{2}}$ by $\frac{N_{1}}{l_{1}} d x_{1} \cdot \frac{N_{2}}{l_{2}} d x_{2}$ and integrating $x_{1}$ from $-l_{1} / 2$ to $l_{1} / 2$ and $x_{2}$ from $-l_{2} / 2$ to $l_{2} / 2$.

For the purpose of finding the corrections mentioned above, it is necessary to write down the results of each of these two integrations. The integration with respect to $x_{1}$ gives the mutual inductance between sheet $s_{1}$ and filament $f_{2}$, which may be called $M_{s_{1} f_{2}}\left(l_{1}, a_{1} ; x_{2}, a_{2} ; \mu\right)$, where

$$
M_{s_{1} f_{2}}\left(l_{1}, a_{1} ; x_{2}, a_{2} ; \mu\right)=\frac{N_{1}}{l_{1}} \int_{-l_{1} / 2}^{l_{1} / 2} d x_{1} M_{f_{1} f_{2}}\left(x_{1}, a_{1} ; x_{2}, a_{2} ; \mu\right),
$$

or since by eq 2

$$
\begin{aligned}
& d x_{1}=-a_{1} \frac{d \alpha_{1}^{\prime}}{\sin ^{2} \alpha_{1}^{\prime}}=a_{1}\left[1-\left(\mu_{1}^{\prime}\right)^{2}\right]^{-\frac{3}{2}} d \mu_{1}^{\prime} \\
& M_{s_{1} f_{2}}=\frac{4 \pi^{2} N_{1} a_{1}}{l_{1}} \sum_{n=1}^{\infty} \frac{P_{n}(\mu)}{n(n+1)}\left[\frac{\left(r_{2}^{\prime}\right)^{n+1}\left(1-\mu_{2}^{\prime 2}\right) P_{n}^{\prime}\left(\mu_{2}^{\prime}\right)}{a_{1}^{n}}\right] \cdot \int_{-\mu_{1}}^{\mu_{1}}\left(1-\mu^{2}\right)^{\frac{n-1}{2}} P_{n}^{\prime}(\mu) d \mu .
\end{aligned}
$$

Now

hence

$$
\left(1-\mu^{2}\right)^{\frac{n-1}{-2}} P_{n}^{\prime}(\mu)=-\frac{1}{(n-1)} \frac{d}{d \mu}\left[\left(1-\mu^{2}\right)^{\frac{n+1}{2}} P_{n-1}^{\prime}(\mu)\right]
$$

$$
\begin{aligned}
\int_{-\mu_{1}}^{\mu_{1}}\left(1-\mu^{2}\right)^{\frac{n-1}{2}} P_{n}^{\prime}(\mu) d \mu & =0, \text { if } n \text { is even } \\
& =2 \mu_{1}, \text { if } n=1 \\
& =\frac{-2\left(1-\mu_{1}^{2}\right)^{s+1} P_{2 s}^{\prime}\left(\mu_{1}\right)}{2 s}, \text { if } n=2 s+1,
\end{aligned}
$$

where $s$ is a positive integer.

This gives

$$
\begin{aligned}
& M_{s_{1} s_{2}}\left(l_{1}, a_{1} ; x_{2}, a_{2} ; \mu\right)=4 \pi \frac{N_{1}}{l_{1}} \pi a_{2}^{2} . \\
& \cdot\left\{\mu \mu_{1}-2\left(1-\mu_{1}^{2}\right) \sum_{n=1}^{\infty} \frac{P_{2 n}^{\prime}\left(\mu_{1}\right) P_{2 n+1}^{\prime}\left(\mu_{2}^{\prime}\right) P_{2 n+1}(\mu)}{2 n(2 n+1)(2 n+2)}\left(\frac{r_{2}^{\prime}}{r_{1}}\right)^{2 n}\right\}
\end{aligned}
$$

The integration with respect to $x_{2}$ gives the mutual inductance between filament $f_{1}$ and current sheet $s_{2}$

$$
\begin{gathered}
d x_{2}=a_{2} \frac{d \mu_{2}^{\prime}}{\left(1-\mu_{2}^{\prime 2}\right)^{\frac{3}{2}}} \\
M_{f_{1} s_{3}}\left(x_{1} a_{1} ; l_{2} a_{2} ; \mu\right)=\frac{N_{2}}{l_{2}} \int_{-l_{2} / 2}^{l_{2} / 2} M_{f_{1} f_{2}}\left(x_{1}, a_{1} ; x_{2} a_{2} ; \mu\right) d x_{2} \\
=\frac{4 \pi^{2} N_{2} a_{2}^{2}}{l_{2}} \sum_{n=1}^{\infty}\left[\frac{\left(1-\mu_{1}^{\prime 2}\right) P_{n}^{\prime}\left(\mu_{1}^{\prime}\right)}{\left(r_{1}^{\prime}\right)^{n}}\right] \frac{P_{n}(\mu) a_{2}^{n}}{n(n+1)} \int_{-\mu_{3}}^{\mu_{2}}\left(1-\mu^{2}\right)^{-\frac{n+2}{2}} P_{n}^{\prime}(\mu) d \mu .
\end{gathered}
$$


Using the relation

gives

$$
\left(1-\mu^{2}\right)^{-\frac{n+2}{2}} P_{n}^{\prime}(\mu)=\frac{1}{n+2} \frac{d}{d \mu}\left[\left(1-\mu^{2}\right)^{-\frac{n}{2}} P_{n+1}^{\prime}(\mu)\right]
$$

$\int_{-\mu_{2}}^{\mu_{3}}\left(1-\mu^{2}\right)^{-\frac{n+2}{2}} P_{n}^{\prime}(\mu) d_{\mu}=0$, if $n$ is

$$
=\frac{2}{2 s+3}\left(1-\mu_{2}^{2}\right)^{-s-\frac{1}{2}} P_{2 s+2}^{\prime}\left(\mu_{2}\right), \text { if } n=2 s+1,
$$

where $s=0,1,2,3 \ldots$.

This gives

$$
\begin{aligned}
M_{f_{1} s_{2}}\left(x_{1}, a_{1} ; l_{2}, a_{2} ; \mu\right)=\frac{8 \pi^{2} N_{2} a_{2}^{2}\left(1-\mu_{1}^{\prime 2}\right)}{l_{2}} & \\
& \cdot \sum_{n=1}^{\infty} \frac{P_{2 n-1}^{\prime}\left(\mu_{1}^{\prime}\right) P_{2 n}^{\prime}\left(\mu_{2}\right) P_{2 n-1}(\mu)}{(2 n-1) 2 n(2 n+1)}\left(\frac{r_{2}}{r_{1}^{\prime}}\right)^{2 n-1}
\end{aligned}
$$

\section{MUTUAL INDUCTANCE BETWEEN TWO TURNS OF WIRE}

Let $M_{f_{2} w_{1}}$ denote the mutual inductance between the filament, $f_{2}$ and a wire, $w_{1}$, whose section is symmetrical with respect to its center of gravity, $\left(x_{1}, \bar{a}_{1}\right)$, so that if $d S_{1}=\mathrm{d} x_{1}^{\prime} d a_{1}^{\prime}$ the integrals over the section of $\left(x_{1}^{\prime}-x_{1}\right),\left(x_{1}^{\prime}-x_{1}\right)^{3},\left(a_{1}^{\prime}-\bar{a}_{1}\right)$, and $\left(a_{1}^{\prime}-\bar{a}_{1}\right)^{3}$ are zero, as would be the case for squares or circles. If $\frac{\rho_{1}}{2}$ denotes the radius of gyration of this section, so that

$$
\frac{\rho_{1}^{2}}{4}=\frac{1}{S_{1}} \iint\left(x_{1}^{\prime}-x_{1}\right)^{2} d S_{1}=\frac{1}{S_{1}} \iint\left(a_{1}^{\prime}-\bar{a}_{1}\right)^{2} d S_{1}
$$

then, in the case of round wire, $\rho_{1}$ is just the radius of the wire.

The current density, $i_{1}$, with unit current in the turn, is such that

and

$$
\int \mathcal{S} i_{1} d x_{1}^{\prime} d a_{1}^{\prime}=\int \mathcal{S} i_{1} d S_{1}=1
$$

$$
M_{f_{2} v_{1}}\left(x_{1}, \bar{a}_{1} ; x_{2} a_{2} ; \mu\right)=\int \mathcal{S} M_{f_{1} f_{2}}\left(x_{1}^{\prime}, a_{1}^{\prime} ; x_{2} a_{2} ; \mu\right) i_{1} d x_{1}^{\prime} d a_{1}^{\prime}=\int \mathcal{S} M_{f_{1} r_{2}} i_{1} d S_{1}
$$

If the ratio of any linear dimension of the wire section, $\mathrm{S}_{1}$, to the mean radius, $\bar{a}_{1}$, of the turn is so small that terms of higher order than the third in this ratio are negligible compared to unity, then the "natural" distribution of current density (proportional to $1 / a_{1}^{\prime}$ ) may be written

$$
i_{1}=\frac{1}{S_{1}}\left\{1-C_{1}\left(\frac{a_{1}^{\prime}-\bar{a}_{1}}{\bar{a}_{1}}\right)+\frac{C_{2}}{\bar{a}_{1}^{2}}\left[\left(a_{1}^{\prime}-\bar{a}_{1}\right)^{2}-\frac{\rho_{1}^{2}}{4}\right]-C_{3}\left(\frac{\bar{a}_{1}-a_{1}}{\bar{a}_{1}}\right)^{3}\right\},
$$

where $C_{1}=C_{2}=C_{3}=1$. For the uniform distribution we place $C_{1}=$ $C_{2}=C_{3}=0$. To the same precision, the expansion may be used

$$
M_{f_{1} f_{2}}\left(x_{1}^{\prime}, a_{1}^{\prime} ; x_{2}, a_{2} ; \mu\right)=\left[1+\phi+\frac{\phi^{2}}{2}+\frac{\phi^{3}}{6}\right] M_{f_{1} f_{2}}\left(x_{1}, \bar{a}_{1} ; x_{2}, a_{2} ; \mu\right),
$$

where $\phi$ is the operator $\left(x^{\prime}-x_{1}\right) D_{x^{1}}+\left(a_{1}^{\prime}-\bar{a}_{1}\right) D_{\bar{a}_{1}}$. 
Multiplying this by $i_{1} d S_{1}$ and integrating gives

$$
M_{r_{2} w_{1}}=\left\{1+\frac{\rho_{1}^{2}}{8}\left(D_{x_{1}}^{2}+D_{\bar{a}_{1}}^{2}-\frac{2 C_{1}}{\bar{a}_{1}} D_{\bar{a}_{1}}\right)\right\} M_{r_{1} f_{2}}\left(x_{1}, \bar{a}_{1} ; x_{2}, a_{2} ; \mu\right)
$$

or by eq $4 \mathrm{a}$

$$
M_{r_{3} x_{1}}=\left\{1+\frac{\rho_{1}^{2}}{8 \bar{a}_{1}} \cdot\left(1-2 C_{1}\right) D_{\bar{a}_{1}}\right\} M_{r_{1} r_{i}} \text {. }
$$

which may be written (since $C_{1}=0$ for uniform current and $C_{1}=1$ for the "natural" distribution) $M_{f_{2} \nu_{1}}=\left(1 \pm \frac{\rho_{2}^{1}}{8 \bar{a}_{1}} D_{\bar{a}_{1}}\right) M_{f_{1} f_{2}}$, the upper sign being used when the current distribution is uniform, the lower when it is the "natural" one. Hence the mutual inductance, $M_{v v_{1} w_{3}}$, between two wires is

$$
M_{w_{1} w_{2}}=M_{f_{1} r_{2}} \pm \frac{1}{8}\left[\frac{\rho_{1}^{2}}{\bar{a}} D_{\bar{a}_{1}}+\frac{\rho_{1}^{2}}{\bar{a}_{2}} D_{\bar{a}_{2}}\right] M_{f_{1}^{\prime},}
$$

It is similarly found that the mutual inductance, $M_{t_{1} t_{1}}$, between two tapes of radii $\bar{a}_{1}$ and $\bar{a}_{2}$, one extending from $x_{1}-\frac{l_{1}}{2 N_{1}}$ to $x_{1}+\frac{l_{1}}{2 N_{1}}$, the other from $x_{2}-\frac{l_{2}}{2 N_{2}}$ to $x_{2}+\frac{l_{2}}{2 N_{2}}$, is

$$
M_{t_{1} l_{3}}=M_{f_{1} f_{3}}+\frac{1}{24}\left[\left(\frac{l_{1}}{N_{1}}\right)^{2} D_{x_{1}}^{2}+\left(\frac{l_{2}}{N_{2}}\right)^{2} D_{x_{3}}^{2}\right] M_{f_{1} r_{3}}
$$

so that eq 6 a may be written

$$
M_{w_{1} w_{3}}=M_{t_{1} t_{2}} \pm \frac{1}{8}\left(\frac{\rho_{1}^{2}}{\bar{a}_{1}^{1}} D_{\bar{a}_{1}}+\frac{\rho_{2}^{2}}{\bar{a}_{2}} D_{\bar{a}_{2}}\right) M_{f_{1} f_{3}}-\frac{1}{24}\left[\frac{l_{1}^{2}}{N_{1}^{2}} D_{x_{1}}^{2}+\frac{l_{2}^{2}}{N_{2}^{2}} D_{x_{2}}^{2}\right] M_{f_{1} f_{3}}
$$

If the wires are now given a small translation, $u_{x}(x)$, in the direction of their axis, and if their mean radius, $\bar{a}_{t}$ is given a small increase, $u_{a}\left(x_{1}\right)$, then if $u_{x}^{2}$ and $u_{a}^{2}$ are negligible, eq $6 \mathrm{c}$ becomes

$$
\begin{aligned}
& M_{v_{1} v_{\mathrm{g}}}\left(x_{1}, \bar{a}_{1} ; x_{2}, \bar{a}_{2} ; \mu\right)=M_{t_{1_{1}} t_{2}}\left(x_{1}, \bar{a}_{1} ; x_{2}, \bar{a}_{2} ; \mu\right)+ \\
& +\left\{ \pm \frac{1}{8}\left(\frac{\rho_{1}^{2}}{\bar{a}_{1}} D_{\bar{a}_{1}}+\frac{\rho_{2}^{2}}{\bar{a}_{2}} D_{\bar{a}_{2}}\right)+u_{x}\left(x_{1}\right) D_{x_{1}}+u_{a}\left(x_{1}\right) D_{\bar{a}_{1}}+u_{x}\left(x_{2}\right) D_{x_{3}}+u_{a}\left(x_{2}\right) D_{\bar{a}_{3}}\right. \\
& \left.-\frac{1}{24}\left(\frac{l_{1}^{2}}{N_{1}^{2}} D_{x_{1}}^{2}+\frac{l_{2}^{2}}{N_{2}^{2}} D_{x_{2}}^{2}\right)\right) M_{f_{1} \gamma_{2}}\left(x_{1}, \bar{a}_{1} ; x_{2}, \bar{a}_{2} ; \mu\right)
\end{aligned}
$$

\section{MUTUAL INDUCTANCE OF THE COILS}

This is obtained by multiplying eq. 7 by $\frac{N_{1}}{l_{1}} d x_{1} \cdot \frac{N_{2}}{l_{2}} d x_{2}$ and integrating $x_{1}$ from $-\frac{l_{1}}{2}$ to $\frac{l_{1}}{2}$ and $x_{2}$ from $-\frac{l_{2}}{2}$ to $\frac{l_{2}}{2}$. This integration, as 
far as the principal term of eq. 7 is concerned, is exactly equivalent to a summation of all possible pairs of filaments, one of which is always in the first sheet, the other in the second. As to all other terms, it is permissible to replace the double summation by integrations, because they are small, the result holding in a macroscopic sense. It is thus found that

$M=\left[1 \pm \frac{1}{8}\left(\frac{\rho_{1}^{2}}{\bar{a}_{1}} D_{\bar{a}_{1}}+\frac{\rho_{2}^{2}}{\bar{a}_{2}} D_{\bar{a}_{2}}\right)\right] M_{s_{s_{3}} s_{8}}\left(l_{1}, \bar{a}_{1} ; l_{2 \Sigma} \bar{a}_{2} ; \mu\right)$

$+\frac{N_{1}}{l_{1}} \int_{-l_{1} / 2}^{l_{1} / 2}\left[u_{x}\left(x_{1}\right) D_{x_{1}}+u_{a}\left(x_{1}\right) D_{\bar{a}_{1}}\right] \cdot M_{f_{1} s_{2}}\left(x_{1} \bar{a}_{1} ; l_{2}, \bar{a}_{2} ; \mu\right) d x_{1}$

$-\frac{l_{1}}{12 N_{1}}\left[D_{x_{1}} M_{f_{1}} s_{2}\right]_{x_{1}=l_{l} / 2}$

$+\frac{N_{2}}{l_{2}} \int_{-l_{2} / 2}^{l_{2} / 2}\left[u_{x}\left(x_{2}\right) D_{x_{8}}+u_{a}\left(x_{2}\right) D_{\bar{a}_{2}}\right] M_{f_{2} s_{1}}\left(l_{1}, \bar{a}_{1} ; x_{2}, \bar{a}_{2} ; \mu\right) d x_{3}$

$$
-\frac{l_{2}}{12 N_{2}}\left[D_{x_{3}} M_{f_{2} s_{1}}\right]_{x_{3}=l_{2} / 2}
$$

Up to this point, terms of the order of magnitude of $\left(\frac{\rho_{2}}{\bar{a}_{2}}\right)^{4}$ have been neglected in comparison with unity. Thus if $\rho_{2}=0.05 \mathrm{~cm}$ and $\bar{a}_{2}=5 \mathrm{~cm}\left(\frac{\rho_{2}}{\bar{a}_{2}}\right)^{4}=(10)^{-8}$. If we let $\Delta \bar{a}_{2} \equiv \frac{\rho_{2}^{2}}{8 a_{2}}$, then $\frac{\left(\Delta \bar{a}_{2}\right)^{2}}{2}=3(10)^{-9}$, which is also negligible. Hence if the effective radii, $a_{1}$ and $a_{2}$, be defined by

$$
a_{i} \equiv \bar{a}_{i}\left[1 \pm \frac{1}{8}\left(\frac{\rho_{i}}{\bar{a}_{i}}\right)^{2}\right] \text { for } i=1,2,
$$

then, since $f\left(a_{i}\right)=f\left(\bar{a}_{i}\right)+f^{\prime}\left(\bar{a}_{i}\right) \cdot \Delta \bar{a}_{i}+f^{\prime \prime}\left(\bar{a}_{i}\right)\left(\frac{\Delta \bar{a}_{i}}{2}\right)^{2}$ it is evident that eq 8 may be written

$M=M_{s_{1} s_{2}}\left(l_{1}, a_{1} ; l_{2}, a_{2} ; \mu\right)+\frac{N_{1}}{l_{1}} \int_{-l_{1} / 2}^{l_{1} / 2}\left[u_{x}\left(x_{1}\right) D_{x_{1}}+u_{a}\left(x_{1}\right) D_{a_{1}}\right] M_{r_{1} s_{2}}\left(x_{1}, a_{1} ; l_{3}\right.$, $\left.a_{2} ; \mu\right) d x_{1}+\frac{N_{2}}{l_{2}} \int_{-l_{2} / 2}^{l_{2} / 2}\left[u_{x}\left(x_{2}\right) D_{x_{3}}+u_{a}\left(x_{2}\right) D_{a_{3}}\right] M_{s_{1} f_{2}}\left(l_{1}, a_{1} ; x_{2}, a_{2} ; \mu\right) d x_{2}-\frac{l_{1}}{12 N_{1}}$ $\left[D_{x_{1}} M_{f_{1} s_{2}}\right]_{x_{1}=l_{1} / 2}-\frac{l_{2}}{12 N_{2}}\left[D_{x_{1}} M_{f_{3} s_{1}}\right]_{x_{2}=b_{8} / 2}$

Where by eq $5 b$ one finds

$$
\begin{aligned}
-\frac{l_{1}}{12 N_{1}}\left[D_{x_{1}} M_{f_{1} s_{2}}\right]_{x_{1}=l_{1} / 2}= & {\left[\frac{4 \pi N_{1}}{l_{1}} \pi a_{2}^{2} N_{2} \mu_{1}\right] \frac{\left(1-\mu_{1}^{2}\right)\left(1-\mu_{2}^{2}\right) l_{1}^{2}}{12 a_{2}^{2} N_{1}^{2}} } \\
& \cdot \sum_{n=1} C_{n}\left(\mu_{1}\right) C_{n}\left(\mu_{2}\right) P_{2 n-1}(\mu) n\left(n+\frac{1}{2}\right)\left(\frac{r_{2}}{r_{1}}\right)^{2 n}
\end{aligned}
$$


and by eq $5 \mathrm{a}$

$$
\begin{aligned}
-\frac{l_{2}}{12 N_{2}}\left[D_{x_{2}} M_{f_{2} s_{1}}\right]_{x_{2}=l_{2} / 2} & =\left[4 \pi \frac{N_{1}}{l_{1}} \pi a_{2}^{2} N_{2} \mu_{1}\right] \frac{\left(1-\mu_{1}^{2}\right)\left(1-\mu_{2}^{2}\right) l_{2}^{2}}{12 a_{2}^{2} N_{2}^{2}} . \\
& \cdot \sum_{n=1}^{\infty} C_{n}\left(\mu_{1}\right) C_{n}\left(\mu_{2}\right) P_{2 n+1}(\mu) n\left(n+\frac{1}{2}\right)\left(\frac{r_{2}}{r_{1}}\right)^{2 n} .
\end{aligned}
$$

\section{TORQUE INCLUDING THE FIRST FOUR CORRECTIONS}

When the coils are at right angles to each other, $\mu=0$ and the torque is $T=\left(D_{\mu} M\right)_{\mu=0}$, so that

$$
\begin{aligned}
T=T_{z_{1} s_{2}} & +\frac{N_{1}}{l_{1}} \int_{-l_{1} / 2}^{l_{1} / 2}\left[u_{x}\left(x_{1}\right) D_{x_{1}}+u_{a}\left(x_{1}\right) D_{a_{1}}\right] T_{f_{1} s_{2}}\left(x_{1}, a_{1}\right) d x_{1} \\
& +\frac{N_{2}}{l_{2}} \int_{-l_{2} / 2}^{l_{2} / 2}\left[u_{x}\left(x_{2}\right) D_{x_{2}}+u_{a}\left(x_{2}\right) D_{a_{3}}\right] T_{s_{1} f_{2}}\left(x_{2}, a_{2}\right) d x_{2},
\end{aligned}
$$

where, by eq 1

$$
\begin{gathered}
T_{s_{1} s_{2}}=\left(4 \pi \frac{N_{1}}{l_{1}}\right) \pi a_{2}^{2} N_{2} \mu_{1}\left(1+S+S_{1}\right) \\
S=\left(1-\mu_{1}^{2}\right) \sum_{n=1}^{\infty}(-1)^{n+1}\left(\frac{r_{2}}{r_{1}}\right)^{2 n} C_{n}\left(\mu_{1}\right) C_{n+1}\left(\mu_{2}\right)\left(n+\frac{1}{2}\right) A_{n},
\end{gathered}
$$

where$$
A_{n}=\frac{\Gamma\left(n+\frac{1}{2}\right)}{\sqrt{\pi} \Gamma n+1}=\frac{1 \cdot 3 \cdot 5 \cdot \ldots(2 n-1)}{2 \cdot 4 \cdot 6 \cdot \ldots 2 n}
$$$$
S_{1}=\frac{\left(1-\mu_{1}^{2}\right)\left(1-\mu_{2}^{2}\right)}{6 a_{2}^{2}} \sum_{n=1}^{\infty}(-1)^{n+1}\left(\frac{r_{2}}{r_{1}}\right)^{2 n} C_{n}\left(\mu_{1}\right) C_{n}\left(\mu_{2}\right) n\left(n+\frac{1}{2}\right) A_{n} \text {. }
$$$$
\left[n \frac{l_{1}^{2}}{N_{1}^{2}}-\left(n+\frac{1}{2}\right) \frac{l_{2}^{2}}{N_{2}^{2}}\right]
$$

Also, $T_{f_{1} s_{2}}\left(x_{1}, a_{1}\right)=\left(D_{\mu} M_{f_{1} s_{2}}\right)_{\mu=0}$ is the torque exerted by filament $f_{1}$ at $x_{1}$ (with unit current) upon the current sheet $s_{2}$ (also carrying unit current).

$$
\begin{aligned}
\frac{N_{1}}{l_{1}} T_{f_{1} s_{2}}\left(x_{1}, a_{1}\right)=\left(4 \pi \frac{N_{1}}{l_{1}} \pi a_{2}^{2} N_{2}\right) \cdot \frac{2\left(1-\mu_{1}{ }^{2}\right)}{l_{2}} \sum_{1}^{\infty} & (-1)^{n+1}\left(\frac{r_{2}}{r_{1}^{\prime}}\right)^{2 n-1} \\
& \cdot \frac{P_{2 n-1}^{\prime}\left(\mu_{1}^{\prime}\right) P_{2 n}^{\prime}\left(\mu_{2}\right)}{(2 n-1)(2 n+1)} \cdot A_{n}
\end{aligned}
$$

Or, since $\mu_{1}^{\prime}=\frac{x_{1}}{r_{1}^{\prime}}$ and $r_{1}^{\prime}=\sqrt{x_{1}^{2}+a_{1}^{2}}$ and

$$
D_{x}\left[\frac{\left(1-\mu^{2}\right) P_{2 n-1}^{\prime}(\mu)}{r^{2 n-1}(2 n-1)}\right]=-\frac{\left(1-\mu^{2}\right) P_{2 n}^{\prime}(\mu)}{r^{2 n}},
$$


we find the following expressions by which $D_{x_{1}} T_{f_{1} s_{2}}$ and $D_{a_{1}} T_{f_{1} s_{2}}$ may be computed for use in the graphical or numerical evaluation of the integrals, in eq 11 when the variations $u_{x}$, and $u_{a}$ are known.

$$
\begin{array}{r}
\frac{N_{1}}{l_{1}} D_{x_{1}} T_{f_{1} s_{2}}=-\left(4 \pi \frac{N_{1}}{l_{1}} \pi a_{2}^{2} N_{2}\right) \cdot \frac{2\left(1-\mu_{1}^{\prime 2}\right)}{l_{2} r_{2}} \sum_{1}^{\infty}(-1)^{n+1}\left(\frac{r_{2}}{r_{1}^{\prime}}\right)^{2 n} . \\
P_{2 n}^{\prime}\left(\mu_{1}^{\prime}\right) P_{2 n}^{\prime}\left(\mu_{2}\right) \frac{A_{n}}{2 n+1},
\end{array}
$$

and since

$$
D_{a}\left[\frac{\left(1-\mu^{2}\right) P_{2 n}^{\prime}(\mu)}{r^{2 n-1}(2 n-1)}\right]=\frac{\sqrt{1-\mu^{2}} 2 n P_{2 n}(\mu)}{r^{2 n}}=-D_{x}\left[\frac{\sqrt{1-\mu^{2}} P_{2 n-1}(\mu)}{r^{2 n-1}}\right]
$$

$\frac{N_{1}}{l_{1}} D_{a_{1}} T_{\rho_{1} s_{2}}=\left(4 \pi \frac{N_{1}}{l_{1}} \pi a_{2}^{2} N_{2}\right) \cdot \frac{2 \sqrt{1-\mu_{1}^{\prime 2}}}{l_{2} r_{2}} \sum_{1}^{\infty}(-1)^{n+1}\left(\frac{r_{2}}{r_{1}}\right)^{2 n}$.

which may also be written

$$
P_{2 n}\left(\mu_{1}^{\prime}\right) P_{2 n}^{\prime}\left(\mu_{2}\right) \cdot \frac{2 n}{2 n+1} A_{n}
$$

$$
\begin{array}{r}
\frac{N_{1}}{l_{1}} D_{a_{1}} T_{f_{1} s_{2}}=-\left(4 \pi \frac{N_{1}}{l_{1}} \pi a_{2}^{2} N_{2}\right)_{l_{2}}^{2} D_{x_{1}}\left\{\sqrt{1-\mu_{1}^{\prime 2}} \sum_{1}^{\infty}(-1)^{n+1}\left(\frac{r_{2}}{r_{1}^{\prime}}\right)^{2 n-1} .\right. \\
\left.P_{2 n-1}\left(\mu_{1}^{\prime}\right) P_{2 n}^{\prime}\left(\mu_{2}\right) \frac{A_{n}}{2 n+1}\right\}
\end{array}
$$

The torque exerted upon filament $f_{2}$ at $x_{2}$ by current sheet $s_{1}$ is $T_{s_{1} f_{2}}$ $=\left(D \mu M_{s_{1} f_{2}}\right)_{\mu=0}$, so that

$$
\begin{aligned}
& \frac{N_{2}}{l_{2}} T_{s_{1} f_{2}}\left(x_{2}, a_{2}\right)=\left(4 \pi \frac{N_{1}}{l_{1}} \pi a_{2}^{2} N_{2}\right)\left\{\frac{\mu_{1}}{l_{2}}+\frac{2\left(1-\mu_{1}^{2}\right)}{l_{2}} \sum_{1}^{\infty}(-1)^{n+1}\left(\frac{r_{2}^{\prime}}{r_{1}}\right)^{2 n} .\right. \\
& \left.\frac{P_{2 n}^{\prime}\left(\mu_{1}\right) P_{2 n+1}^{\prime}\left(\mu_{2}^{\prime}\right)}{2 n(2 n+2)} \cdot A_{n}\right\} \\
& =\left(4 \pi \frac{N_{1}}{l_{1}} \pi N_{2}\right)\left\{\frac{\mu_{1} a_{2}^{2}}{l_{2}}+\frac{2\left(1-\mu_{1}^{2}\right)}{l_{2}} \sum_{1}^{\infty}(-1)^{n+1}\left[\frac{\left(r_{2}^{\prime}\right)^{2 n+2}\left(1-\mu_{2}^{\prime 2}\right) P_{2 n+1}^{\prime}\left(\mu_{2}^{\prime}\right)}{(2 n+2)}\right]\right. \\
& \left.\frac{P_{2 n}^{\prime}\left(\mu_{1}\right) A_{n}}{2 n r_{1}^{2 n}}\right\},
\end{aligned}
$$

where

$$
\mu_{2}^{\prime}=\frac{x_{2}}{r_{2}^{\prime}} \text { and } r_{2}^{\prime}=\sqrt{x_{2}^{2}+a_{2}^{2}}
$$

Since

$$
D_{x}\left[\frac{r^{2 n+2}\left(1-\mu^{2}\right) P_{2 n+1}^{\prime}(\mu)}{2 n+2}\right]=\left(1-\mu^{2}\right) r^{2 n+1} P_{2 n}^{\prime}(\mu),
$$


it is found that

$$
\begin{aligned}
\frac{N_{2}}{l_{2}} D_{x_{2}} T_{\varepsilon_{1} r_{2}}=\left(\frac{4 \pi N_{1}}{l_{1}} \pi a_{2}^{2} N_{2}\right) \frac{2\left(1-\mu_{1}^{2}\right)}{l_{2} r_{1}} \sum_{1}^{\infty}(-1)^{n+1}\left(\frac{r_{2}^{\prime}}{r_{1}}\right)^{2 n-1} & P_{2 n}^{\prime}\left(\mu_{1}\right) P_{2 n}^{\prime}\left(\mu_{2}^{\prime}\right) \frac{A_{n}}{2 n}
\end{aligned}
$$

Also since

$$
D_{a}\left[\frac{r^{2 n+2}\left(1-\mu^{2}\right) P_{2 n+1}^{\prime}(\mu)}{2 n+2}\right]=\sqrt{1-\mu^{2}}(2 n+1) r^{2 n+1} P_{2 n}(\mu)
$$

one finds

$$
\begin{aligned}
\frac{N_{2}}{l_{2}} D_{a_{2}} T_{s_{1} t_{2}}=\left(\frac{4 \pi N_{1}}{l_{1}} \pi a_{2}^{2} N_{2}\right)\left\{\frac{2 \mu_{1}}{l_{2} a_{2}}+\frac{2\left(1-\mu_{1}^{2}\right)}{l_{2} a_{2}} \sum_{1}^{\infty}(-1)^{n+1}\left(\frac{r_{2}^{\prime}}{r_{1}}\right)^{2 n} .\right. \\
\left.P_{2 n}^{\prime}\left(\mu_{1}\right) P_{2 n}\left(\mu_{2}^{\prime}\right) \frac{(2 n+1)}{2 n} A_{n}\right\}
\end{aligned}
$$

When the outer coil is relatively long, the inner lies in a region where the axial field of the outer has the practically uniform value $4 \pi \frac{N_{1}}{l_{1}}$. The series $S$, eq $12^{\prime}$, is then very small and $\mu_{1}$ is nearly 1 and the term $S_{1}$, eq $12^{\prime \prime}$, still smaller. This will be the case if $l_{1}=100 \mathrm{~cm}$ and $a_{1}=15 \mathrm{~cm}$, so that $\mu_{1}=0.9578$ and $1-\mu_{1}^{2}=0.0826$ and $r_{1}=52.44 \mathrm{~cm}$. If the inner coil has the radius $a_{2}=5 \mathrm{~cm}$ and length $l_{2}=10 \mathrm{~cm}$, then $\mu_{2}=\frac{1}{\sqrt{2}}$ and $1-\mu_{2}^{2}=\frac{1}{2}$, while $r_{2}=7.07 \mathrm{~cm}$.

Since $r_{1}^{\prime} \geqq a_{1}$ so that $\left(\frac{r_{2}}{r_{1}^{\prime}}\right)^{2}<\left(\frac{r_{2}}{a_{1}}\right)^{2}=\frac{1}{4}$, it is seen that the series 13 will differ from the sum of the first two terms by less than 4 percent. The first two terms of eq 14 give a still better approximation.

To this approximation eq 13 and 14 become

$$
\frac{N_{1}}{l_{1}} T_{f_{1},}=4 \pi \frac{N_{1}}{l_{1}} \pi a_{2}^{2} N_{2} \cdot \frac{a_{1}^{2}}{2\left(x_{1}^{2}+a_{1}^{2}\right)^{\frac{3}{2}}}\left\{1+\frac{3\left(l_{2}^{2}-3 a_{2}^{2}\right)}{16 a_{1}^{2}}\left[\frac{5}{\left(1+\frac{x_{1}^{2}}{a_{1}^{2}}\right)^{2}}-\frac{4}{1+\frac{x_{1}^{2}}{a_{1}^{2}}}\right]\right\}
$$

and

$$
\frac{N_{2}}{l_{2}} T_{s_{1} f_{2}}=4 \pi \frac{N_{1}}{l_{1}} \pi a_{2}^{2} N_{2} \bar{l}_{2}\left\{1+\frac{9\left(4 x_{2}^{2}-a_{2}^{2}\right) a_{1}^{2}}{\left(l_{1}^{2}+4 a_{1}^{2}\right)^{2}}\right\}
$$

Since the small observed displacements, $u_{x}, u_{a}$, cannot be measured with a precision of more than 3 or 4 percent it is evident that the terms within the braces of eq 15 and 16 may be placed equal to unity so that a good approximation for eq 11 is

$$
\begin{aligned}
& T=4 \pi \frac{N_{1}}{l_{1}} \pi a_{2}^{2} N_{2} \mu_{1}\left\{1+S+S_{1}+\frac{2}{l_{2} a_{2}} \int_{-l_{2} / 2}^{l_{2} / 2} u_{a}\left(x_{2}\right) d x_{2}+\right. \\
& \left.\frac{a_{1}}{2 \mu_{1}} \int_{-l_{2} / 2}^{l_{2} / 2}\left[u_{x}\left(x_{1}\right) D_{x_{1}} \frac{a_{1}}{\left(x_{1}^{2}+a_{3}^{2}\right)^{\frac{a}{2}}}-u_{a}\left(x_{1}\right) D_{x_{1}} \frac{x_{1}}{\left(x_{1}^{2}+a_{1}^{2}\right)^{\frac{3}{2}}}\right] d x_{1}\right\}
\end{aligned}
$$

If $u_{a_{2}}\left(x_{2}\right)=\delta a_{2}=$ constant, $u_{a}\left(x_{1}\right)=\delta a_{1}=$ constant and $u_{x}\left(x_{1}\right)=\frac{\delta l_{1}}{l_{1}} \cdot x_{1}$, the 
evaluation of these integrals gives

$$
T=4 \pi \frac{N_{1}}{l_{1}} \pi a_{2}^{2} N_{2} \mu_{1}\left\{1+S-\frac{l_{1}^{2}}{l_{1}^{2}+4 a_{1}^{2}} \cdot \frac{\delta l_{1}}{l_{1}}-\frac{4 a_{1}^{2}}{l_{1}^{2}+4 a_{1}^{2}} \frac{\delta a_{1}}{a_{1}}+\frac{2 \delta a_{2}}{a_{2}}\right\}
$$

The displacement $u_{x}\left(x_{1}\right)=\frac{\delta l_{1}}{l_{1}} x_{1}$ corresponds to an increase in the length, $l_{1}$, without changing the total number, $N_{1}$, of turns, which amounts to increasing the pitch of the windings a constant amount.

A similar displacement, $u_{x}\left(x_{2}\right)=\frac{\delta l_{2}}{l_{2}} x_{2}$, in the second coil would by eq 17 have contributed nothing to the second member of eq 18. The latter may be written

$$
\frac{\delta T}{T}=-\frac{l_{1}^{2}}{l_{1}^{2}+4 a_{1}^{2}} \cdot \frac{\delta l_{1}}{l_{1}}-\frac{4 a_{1}^{2}}{l_{1}^{2}+4 a_{1}^{2}} \frac{\delta a_{1}}{a_{1}}+0 \frac{\delta l_{2}}{l_{2}}+2 \frac{\delta a_{2}}{a_{2}}
$$

These variations would give by eq 12

$$
\begin{aligned}
\frac{\delta T_{s_{1} s_{2}}=-\left[\frac{l_{1}^{2}}{T_{s_{1}, s}}-\frac{l_{1}}{l_{1}^{2}+4 a_{1}^{2}}-\frac{\partial S}{1+S} \frac{\delta l_{1}}{\partial l_{1}}\right]}{\frac{\delta l_{1}}{l_{1}}}-\left[\frac{4 a_{1}^{2}}{l_{1}^{2}+4 a_{1}^{2}}-\frac{a_{1}}{1+S} \frac{\partial S}{\partial a_{1}}\right] \frac{\delta a_{1}}{a_{1}} \\
+\frac{l_{2}}{1+S} \frac{\partial S}{\partial l_{2}} \cdot \frac{\delta l_{2}}{l_{2}}+\left[2+\frac{a_{2}}{1+S} \frac{\partial S}{\partial a_{2}}\right] \frac{\delta a_{2}}{a_{2}}
\end{aligned}
$$

A comparison of eq $18^{\prime}$ and 19 shows (since $\frac{\delta T_{s_{1} s_{2}}}{T_{s_{1} s_{2}}}$ is practically the same as $\frac{\delta T}{T}$ ) that the first approximation eq 17 , which was derived by neglect of all but the first term in the brackets of eq 15 and 16, is compatible with the statement that $S$ is so small that its variations may be neglected in computing the variations of $\delta T$ caused by varying $l_{1}, a_{1}, a_{2}$, and $l_{2}$. If the more precise variation formula eq 19 were desired it could be found by retaining all the terms in eq 15 and 16 . The cylindrical shell upon which the outer solenoid is wound may be slightly compressed by the tension of the wires so that the effective radius, $a_{1}(x)$, of the current sheet may be represented by the parabolic equation

$$
a_{1}\left(x_{1}\right)=a_{1}(0)+\left[a_{1}\left( \pm \frac{l_{1}}{2}\right)-a_{1}(0)\right]\left(\frac{2 x_{1}}{l_{1}}\right)^{2} .
$$

The effective mean radius, $a_{1}$, of the equivalent sheet is then

so that

$$
a_{1}=\frac{2}{l_{1}} \int_{0}^{l_{1} / 2} a_{1}\left(x_{1}\right) d x_{1}=\frac{2}{3} a_{1}(0)+\frac{1}{3} a_{1}\left( \pm \frac{l_{1}}{2}\right),
$$

and

$$
u_{a}\left(x_{1}\right)=a_{1}\left(x_{1}\right)-a_{1}=-\left[a_{1}\left( \pm \frac{l_{1}}{2}\right)-a_{1}(0)\right]\left[\frac{1}{3}-\left(\frac{2 x_{1}}{l_{1}}\right)^{2}\right]
$$

$$
\int_{0}^{l_{1} / 2} u_{a}\left(x_{1}\right) d x_{1}=0 \text {. }
$$


Equation 17, then, gives the correction for this deformation in the form

$$
\frac{\delta T}{T}=-\frac{4 a_{1}\left[a_{1}\left( \pm \frac{l_{1}}{2}\right)-a_{1}(0)\right]}{l_{1}^{2}}\left\{1+\frac{1}{3\left[1+\left(\frac{2 a_{1}}{l_{1}}\right)^{2}\right]}-\sqrt{1+\left(\frac{2 a_{1}}{l_{1}}\right)^{2}} .\right.
$$

In computing this small correction term, $a_{1}$ may be taken as the mean radius defined by eq 21 , where $2 a_{1}(x)$ is the over-all diameter of the coil at $x_{1}$ diminished by the diameter $2 \rho_{1}$ of the wire. This was denoted previously (in section V) by $\bar{a}_{1}$, but there is no need to distinguish between $a_{1}$ and $\bar{a}_{1}$ in computing the small correction, eq 22 . However, this correction is applicable only provided that the term $\mu_{1}(1+S)$ of eq 17 is computed using the "effective" or "electrical" mean radius, $a_{1}$, defined in eq 9 by $a_{1}=\bar{a}_{1}\left(1 \pm \frac{\rho_{1}^{2}}{8 a_{1}^{2}}\right)$ the upper sign being taken if we believe the current distribution is uniform over the section of the wires, the lower sign if it is the natural distribution.

The corrections $(a)$ and $(b)$ mentioned in the section I are represented by this choice of effective radii of the current sheets (eq 9). The corrections $(c)$ and $(d)$ are made by the integrals in eq 17 .

\section{EFFECT OF ERRORS IN CENTERING THE COILS}

The effect upon the torque of an error in centering the outer coil may be found by giving it a constant displacement in the direction of its axis. But if $u_{x}\left(x_{1}\right)=$ constant the corresponding integral in eq 17 vanishes. Similarly, there is a negligible effect in giving the inner coil a constant displacement in its axial direction. Both of these results are aspects of the fact that the inner coil lies in a region where the field of the outer is practically uniform, so that for the same reason it is evident that the only other independent displacement of the inner coil would also be negligible. This is a displacment perpendicular to the plane of the two axes.

\section{EFFECT OF AXIAL COMPONENTS OF CURRENT}

Up to this point the current sheets have been assumed to have only the angular components of current whose surface density per unit length is $\mathrm{N}_{i} / l_{i}$. Each coil is, however, a helix, so that there is an axial component of current whose surface density is $\pm 1 / 2 \pi a$ per unit length, the positive sign being taken when the axial current is in the direction of increasing $x_{i}$ (fig. 1). To see what modification this makes in the torque, we first see what modification must be made in the mutual inductance between the two sheets when their axes are inclined at an angle $\theta=\cos ^{-1} \mu$. To do this we first modify the expression for the mutual inductance between filament No. 2 whose trace is $A_{2}^{\prime} C_{2}^{\prime} B_{2}^{\prime}$ in figure 1 and filament No. 1 trace $A_{1}^{\prime} C_{1}^{\prime} B_{1}^{\prime}$, remembering that these now have the additional axial components of current.

Let $A^{\circ}(x y z)$ be the vector potential at any point $(x y z)$ which is due 
to the angular component of current $\frac{N_{1}}{l_{1}} d x_{1}$ in No. 1 and let $A(x y z)$ be that due to the axial current $\pm \frac{d x_{1}}{2 \pi a_{1}}$ in No. 1 .

The angular component of current in filament No. 2 is $\frac{N_{2}}{l_{2}} d x_{2}$, its axial component is $\pm \frac{d x_{2}}{2 \pi a_{2}}$. Hence the mutual electrokinetic energy between these filaments is

$$
\text { M. } F_{6}=\frac{N_{2} d x_{2}}{l_{2}} \int d s_{2}\left[A_{s_{2}}^{0}+A_{s_{2}}\right]+\frac{d x_{2}}{2 \pi a_{2}} \int\left[A_{n_{2}}^{0}+A_{n_{2}}\right] d s_{2},
$$

where the line integrals are taken around circle No. 2 (right handedly), $A_{s_{2}}$ is the tangential component of the vector $A$ and $A_{n_{3}}$ its component normal to the plane of the circle. Only the last three terms need be considered here, since the first, $\int A_{s_{2}}^{0} d s_{2}$, leads to the mutual inductance, $M_{s_{1}, s}$, already found. The second is a line integral of the tangential component of the vector $A$ and by Stokes' theorem may be written $\frac{N_{2} d x_{2}}{l_{2}} \iint H_{n_{3}} d S_{2}$, the integral being taken over the area of the circle No. 2, where $\mathrm{H}=$ curl $A=$ magnetic vector due to the axial component of current in filament No. 1.

Since the vector $A$ at any point is in the direction of the axis of circle No. 1 it is evident that $A_{n_{3}}=A_{x_{1}} \cos \theta=\mu A_{x_{1}}$, so that the fourth integral is $\pm \frac{d x_{2}}{2 \pi a_{2}} \mu \int A_{x_{1}} d s_{2}$.

Hence the mutual energy is (omitting the first term)

$$
\text { M. E. }=\frac{N_{2}}{l_{2}} d x_{2} \iint H_{n_{2}} d S_{2} \pm \frac{d x_{2}}{2 \pi a_{2}} \int A_{n_{3}}^{0} d s_{2} \pm \frac{d x_{2}}{2 \pi a_{2}} \mu \int A_{x_{1}} d s_{2}
$$

Now, if $x, r, \phi$ are cylindrical coordinates of any point, the axis being that of the circle 1, then $A_{x_{1}}$ is a function of $x$ and $r$ only, $r$ being the distance of the point from the axis. Since $H=\operatorname{curl} A$, the cylindrical components of $H$ are $H_{x} \equiv H_{r} \equiv 0, H_{\phi}=-D_{r} A_{x_{1}}(x, r)$.

Hence $\iint H_{n} d S_{2}=0$, since the value of $H_{n}$ has equal and opposite values at the two points in the area $S_{2}$, whose coordinates are $x, r, \phi$, and $x, r,-\phi$. Similarly, the second integral of equation 23 vanishes, since the only nonvanishing cylindrical component of $A^{0}$ is $A_{\phi}^{0}$, which is a function of $x$ and $r$ only. At two elements of arc of the circle No. 2, whose coordinates are $x, r, \phi$, and $x, r,-\phi$, the values of $A^{0}{ }_{n_{2}}$ cancel.

The expression 23 for the mutual energy then reduces to that between the two axial currents in the filament and may be written

$$
m_{f_{1} f_{3}} d x_{1} d x_{2}= \pm \frac{\mu d x_{2}}{2 \pi a_{2}} \int A_{x_{1}} d s_{2}
$$

Since $A_{x_{1}}$ at any point $x y z$ is given by

$$
A_{x_{1}}= \pm \frac{d x_{1}}{2 \pi a_{1}} \int \frac{d s_{1}}{R}
$$


where $R$ is the distance from $x y z$ to the line element $d s_{1}$ of circle 1 , it is evident that the mutual inductance of the two unit axial currents in the filaments is

$$
m_{f_{1} f_{2}}= \pm \frac{\mu}{2 \pi a_{1} 2 \pi a_{2}} \int d s_{2} \int \frac{d s_{1}}{R},
$$

where $R$ is the distance from $d s_{1}$ to $d s_{2}$, the + sign applying to the case where the axial currents have the same sign, the - where their algebraic signs are different, positive axial current being in the direction of increasing $x_{1}$ or $x_{2}$, as shown in figure 1 .

The double line integral in equation 26 without the factor $\pm \mu$ represents the mutual electrostatic energy of the two circular filaments when they are regarded as unit line charges of electricity.

The contribution of the axial components in the current sheets to their mutual inductance is therefore

$$
m_{\varepsilon_{1} s_{2}}(\mu)=\int_{-l_{2} / 2}^{l_{2} / 2} d x_{2} \int_{-l_{1} / 2}^{l_{1} / 2} d x_{1} m_{f_{1} t_{1}}
$$

and the contribution to the torque is

$$
t_{s_{1} s_{2}}=\left[D_{\mu} m_{s_{1} s_{2}}(\mu)\right]_{\mu=0}
$$

To evaluate the integral $\frac{1}{2 \pi a_{2}} \int A_{x_{1}} d s_{2}$, we note that $A_{x_{1}}$ is a solution of Laplace's equation, which, in rectangular coordinates, is

$$
\left(D_{x}^{2}+D_{y}^{2}+D_{z}^{2}\right) A_{x_{1}}=0 .
$$

Expand $A_{x_{1}}$ by Taylor's theorem in the symbolic form

$$
\begin{aligned}
A_{x_{1}}(x, y, z) & =A_{x_{1}}\left(x_{0}+x-x_{0}, y_{0}+y-y_{0}, z_{0}+z-z_{0}\right) \\
& =e^{\left(x^{\prime} D_{x_{0}}+y^{\prime} D_{s_{0}}+z^{\prime} D_{z_{0}}\right)} A_{x_{1}}\left(x_{0}, y_{0}, z_{0}\right),
\end{aligned}
$$

where $x^{\prime}=x-x_{0}, y^{\prime}=y-y_{0}$ and $z^{\prime}=z-z_{0}$.

It is then found by integration that, if $J_{0}(z)$ is Bessel's function $\frac{1}{2 \pi a_{2}} \int A_{x_{1}}(x, y, z,) d s_{2}=J_{0}\left(a_{2} D_{n_{2}}\right) A_{x_{1}}\left(x_{0}, y_{0}, z_{0}\right)$, where $x_{0}, y_{0}, z_{0}$ are rectangular coordinates of the center of the circle No. 2 and $D_{n_{3}}$ represents the directional derivative in the direction normal to the plane of the circle No. 2. Since we have used $x_{2}$ as the distance $\overline{O C_{2}^{\prime}}$ in figure 1 (not $a$. rectangular coordinate) this becomes

$$
\frac{1}{2 \pi a_{2}} \int A_{x_{1}} d s_{2}=J_{0}\left(a_{2} D_{x_{2}}\right) A_{x_{1}}\left(x_{0}, y_{0}, z_{0}\right),
$$

which holds when $A_{x_{1}}$ is any harmonic scalar point function.

Now

$$
A_{x_{1}}\left(x_{0}, y_{0}, z_{0}\right)= \pm \frac{d x_{1}}{2 \pi a_{1}} \int \frac{d s_{1}}{R}
$$


where $R$ is the distance from $x_{0}, y_{0}, z_{0}$ to the element $d s_{1}$ of the circular arc of No. 1, so that $A_{x_{1}}$ at any point is $\pm d x_{1}$ times the electrostatic potential at that point due to a unit circular line charge (the circle No. 1). It may be put in the form

$$
A_{x_{1}}\left(x_{0}, y_{0}, z_{0}\right)= \pm d x_{1} \int_{0}^{\infty} e^{-s\left|x_{0}-x_{1}\right|} J_{0}\left(a_{1} s\right) J_{0}(r s) d s
$$

where $r$ is the distance of $x_{0}, y_{0}, z_{0}$ from the axis of the circle No. 1 . This may also be written, symbolically, $A_{x_{1}}\left(x_{0}, y_{0}, z_{0}\right)= \pm d x_{1} J_{0}\left(a_{1} D_{x_{1}}\right)$ $\frac{1}{R_{12}}$, where $R_{12}$ is the distance between the centers of the two circles.

Now

$$
\frac{1}{R_{12}}=\frac{1}{\sqrt{x_{1}^{2}-2 x_{1} x_{2} \cos \theta+x_{2}^{2}}}=\sum_{n=0}^{\infty} \frac{x_{2}^{n}}{x_{1}^{n+1}} P_{n}(\mu) \text {, if } x_{1}>\left|x_{2}\right|
$$

Hence

$$
\begin{aligned}
& A_{x_{1}}\left(x_{0}, y_{0}, z_{0}\right) \\
& = \pm d x_{1} \sum_{s=0}^{\infty} \frac{(-1)^{s}\left(\frac{a_{1}}{2}\right)^{2 s}}{\Gamma(s+1) \Gamma(s+1)} D_{x_{1}}^{2 s} \sum_{n=0}^{\infty} \frac{x_{2}^{n}}{x_{1}^{n+1}} P_{n}(\mu) \\
& = \pm \frac{d x_{1}}{x_{1}} \sum_{s=0}^{\infty} \frac{(-1)^{s}\left(\frac{a_{1}}{2 x 1}\right)^{2 s}}{\Gamma(s+1) \Gamma(s+1)} \sum_{n=0}^{\infty}\left(\frac{x_{2}}{x_{1}}\right)^{n} \frac{\Gamma(n+2 s+1)}{\Gamma(n+1)} P_{n}(\mu) \\
& = \pm \frac{d x_{1}}{x_{1}} \sum_{n=0}^{\infty}\left(\frac{x_{2}}{x_{1}}\right)^{n} \frac{P_{n}(\mu)}{\Gamma(n+1)} \sum_{s=0}^{\infty}(-1)^{s}\left(\frac{a_{1}}{2 x_{1}}\right)^{2 s} \frac{\Gamma(2 s+n+1)}{\Gamma(s+1) \Gamma(s+1)} \\
& = \pm \frac{d x_{1}}{x_{1} \sqrt{\pi}} \sum_{n=0}^{\infty}\left(\frac{2 x_{2}}{x_{1}}\right)^{n} \frac{P_{n}(\mu)}{\Gamma(n+1)} \sum_{s=0}^{\infty}(-1)^{s}\left(\frac{a_{1}}{x_{1}}\right)^{2 s} \frac{\Gamma\left(s+\frac{n+1}{2}\right)}{\Gamma\left(s+\frac{n+2}{2}\right)} \\
& = \pm \frac{d x_{1}}{x_{1}} \sum_{0}^{\infty}\left(\frac{x_{2}}{x_{1}}\right)^{n} P_{n}(\mu) F\left(\frac{n}{2}+1, \frac{n}{2}+\frac{1}{2}, 1 ;-\frac{a_{1}^{2}}{x_{1}^{2}}\right),
\end{aligned}
$$

so that

$$
A_{x_{1}}\left(x_{0}, y_{0}, z_{0}\right)= \pm d x_{1} \sum_{0}^{\infty} \frac{x_{2}^{n} P_{n}\left(\mu_{1}^{\prime}\right) P_{n}(\mu)}{\left(r_{1}^{\prime}\right)^{n+1}} \text { since } r_{1}^{\prime}>\left|x_{2}\right|
$$

The derivation assumes $x_{1}$ positive, but by repeating the above argument, when $x_{1}<0$ but $\left|x_{1}\right|>\left|x_{2}\right|$, it is found that eq 30 remains valid for all real values of $x_{1}$ and $x_{2}$ for which $r_{1}^{\prime}>\left|x_{2}\right|$ 
Now

$$
\begin{aligned}
& J_{0}\left(a_{2} D_{x_{2}}\right) \sum_{0}^{\infty} \frac{x_{2}^{n} P_{n}\left(\mu_{1}^{\prime}\right) P_{n}(\mu)}{\left(r_{1}^{\prime}\right)^{n+1}}=\sum_{s=0}^{\infty} \frac{(-1)^{s}\left(\frac{a_{2}}{2}\right)^{2 s}}{\Gamma(s+1) \Gamma(s+1)} D_{x_{2}}^{2_{2}} \sum_{n=0}^{\infty} \frac{x_{2}^{n} P_{n}\left(\mu_{1}^{\prime}\right) P_{n}(\mu)}{\left(r_{1}^{\prime}\right)^{n+1}} \\
& =\sqrt{\pi} \sum_{s=0}^{\infty} \frac{(-1)^{s}\left(\frac{a_{2}}{2}\right)^{2 s}}{\Gamma(s+1) \Gamma(s+1)} \sum_{n=0}^{\infty}\left(\frac{x_{2}}{2}\right)^{n-2 s} \frac{\Gamma(n+1) P_{n}\left(\mu_{1}^{\prime}\right) P_{n}(\mu)}{\Gamma\left(\frac{n+1}{2}-s\right) \Gamma\left(\frac{n+2}{2}-s\right)\left(r_{1}^{\prime}\right)^{n+1}} \\
& =\sqrt{\pi} \sum_{n=0}^{\infty}\left(\frac{x_{2}}{2}\right)^{n} \frac{P_{n}\left(\mu_{1}^{\prime}\right) P_{n}(\mu) \Gamma(n+1)}{\left(r_{1}^{\prime}\right)^{n+1}} \sum_{s=0}^{\infty}(-1)^{s}\left(\frac{a_{2}}{x_{2}}\right)^{2 s} \\
& =\sum_{n=0}^{\infty} \frac{x_{2}^{n}}{\left(r_{1}^{\prime}\right)^{n+1}} P_{n}\left(\mu_{1}^{\prime}\right) P_{n}(\mu) F\left(\frac{-n}{2}, \frac{1}{2}-\frac{n}{2}, 1 ;-\frac{a_{2}^{2}}{x_{2}^{2}}\right) \\
& =\sum_{n=0}^{\infty} \frac{r_{2}^{\prime n}}{\left(r_{1}^{\prime}\right)^{n+1}} P_{n}\left(\mu_{1}^{\prime}\right) P_{n}\left(\mu_{2}^{\prime}\right) P_{n}(\mu)
\end{aligned}
$$

The mutual inductance of the two current filaments with unit axial currents is therefore, by eq $26,29,30$, and the preceding equation

$$
m_{f_{1} f_{2}}= \pm \mu \sum_{n=0}^{\infty} \frac{r_{2}^{\prime n}}{\left(r_{1}^{\prime}\right)^{n+1}} P_{n}\left(\mu_{1}^{\prime}\right) P_{n}\left(\mu_{2}^{\prime}\right) P_{n}(\mu)=\frac{ \pm \mu}{2 \pi a_{1} 2 \pi a_{2}} \int d s_{2} \int \frac{d s_{1}}{R}
$$

which converges for $r_{2}^{\prime}<r_{1}^{\prime}$, which is always the case if $f_{1}$ is in sheet No. 1 and $f_{2}$ in sheet No. 2 .

\section{Hence}

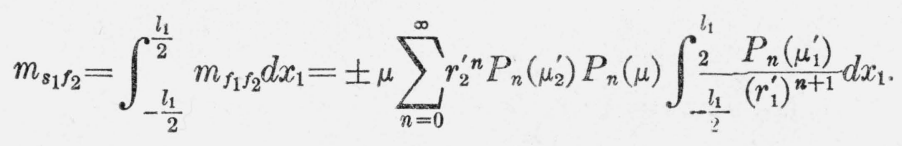

Since $\frac{P_{n}(\mu)}{r^{n+1}}=-D_{x} \frac{P_{n-1}(\mu)}{n r^{n}}$ if $n>0$

$$
=D_{x} \log \left(x+\sqrt{x^{2}+a_{2}}\right) \text { if } n=0,
$$


the integration gives

$$
m_{s_{1} f_{2}}= \pm 2 \mu\left\{\log \left(\frac{l_{1}+\sqrt{l_{1}^{2}+4 a_{1}^{2}}}{2 a_{1}}\right)-\sum_{n=1}^{\infty}\left(\frac{r_{2}^{\prime}}{r_{1}}\right)^{2 n} \frac{P_{2 n-1}\left(\mu_{1}\right) P_{2 n}\left(\mu_{2}^{\prime}\right) P_{2 n}(\mu)}{2 n}\right\} \text {. }
$$

Then

$m_{s_{1} s_{2}}=\int_{-\frac{l_{2}}{2}}^{\frac{l_{2}}{2}} m_{s_{1} f_{2}} d x_{2}$ and since $r^{2 n} P_{2 n}(\mu)=D_{x}\left[\frac{r^{2 n+1} P_{2 n+1}(\mu)}{2 n+1}\right]$

$m_{s_{1} s_{2}}(\mu)= \pm 2 \mu\left\{l_{2} \log \left(\frac{l_{1}+\sqrt{l_{1}^{2}+4 a_{1}^{2}}}{2 a_{1}}\right)-2 r_{2} \sum_{n=1}^{\infty}\left(\frac{r_{2}}{r_{1}}\right)^{2 n}\right.$

so that the torque is

$$
\left.\frac{P_{2 n-1}\left(\mu_{1}\right) P_{2 n+1}\left(\mu_{2}\right) P_{2 n}(\mu)}{2 n(2 n+1)}\right\}
$$

$$
\begin{aligned}
t_{s_{1} \varepsilon_{2}}= \pm 2 l_{2}\left\{\log \left(\frac{l_{1}+\sqrt{l_{1}^{2}+4 a_{1}^{2}}}{2 a_{1}}\right)+\frac{1}{\mu_{2}} \sum_{n=1}^{\infty}(-1)^{n+1}\left(\frac{r_{2}}{r_{1}}\right)^{2 n}\right. \\
\\
\left.\quad \frac{P_{2 n-1}\left(\mu_{1}\right) P_{2 n+1}\left(\mu_{2}\right)}{2 n(2 n+1)} \cdot A_{n}\right\}
\end{aligned}
$$

For the case here considered the series is negligible so that we may take

$$
\begin{gathered}
t_{s_{1} s_{2}}= \pm 2 l_{2} \log \left(\frac{l_{1}+\sqrt{l_{1}^{2}+4 a_{1}^{2}}}{2 a_{1}}\right), \\
\begin{array}{c}
\text { ( if axial components of current } \\
\text { have the same algebraic sign) }
\end{array}
\end{gathered}
$$

which must be added to the expression 17 for the torque. For the case noted, $l_{1}=100 \mathrm{~cm}, a_{1}=15 \mathrm{~cm}$, and $l_{2}=2 a_{2}=10 \mathrm{~cm}$, so that $t_{s_{1} s_{2}}= \pm 27.2$.

The principal part of the torque is $T_{s_{1} s_{2}}=4 \pi \frac{N_{1}}{l_{1}} \pi a_{2}^{2} N_{2}$, so that if $N_{1}=1,000$ and $N_{2}=100, T_{s_{1} s_{2}}=\pi^{2} 10^{5}=10^{6}$, so that the correction $t_{s_{1} s_{2}}$ amounts to 27 parts in a million.

\section{EFFECT OF LEAD-IN WIRES}

Consider the inner coil with its axis vertical, the coil being balanced on a knife-edge which is perpendicular to the plane of figure 1 . If the lead-in wires to the inner coil lie in the vertical plane containing the knife-edge, the electromagnetic forces upon them would contribute nothing to the torque about that knife-edge. The force on any element of these wires would lie in the vertical plane containing the knife-edge, since the magnetic field of the outer coil is practically 
uniform. It remains to compute the effect of the return current in the lead wire of the outer coil. This will be done for the case where the return wire is just outside the solenoid and in the plane of the paper of figure 1, either above the solenoid or below it. The unit current in this wire will have an opposite algebraic sign to that of the axial unit current in coil No. 1. Its vector potential at any point will reduce to the single $x_{1}$-component: $A_{x_{1}}$ parallel to the axis of coil 1 . Let $m_{l_{1} s_{2}}$ and $m_{l_{1} s_{2}}$ denote the mutual inductance between this lead wire and filament No. 2, sheet No. 2, respectively. The mutual energy between filament No. 2 and this wire will be of the same form as eq 23 without the term $A^{0}$, and the surface integral of $H_{n_{2}}$ will also vanish because the lead-in wire lies in the plane of the common axes of the coils, so that the mutual energy is

$$
m_{l_{1} f_{2}} d x_{2}= \pm \mu d x_{2} \frac{1}{2 \pi a_{2}} \int A_{x_{1}} d s_{2}= \pm \mu d x_{2} J_{0}\left(a_{2} D_{x_{z}}\right) A_{x_{1}}\left(x_{0}, y_{0}, z_{0}\right),
$$

where the choice of signs is the same as in eq 23, positive if the axial current in $f_{2}$ is upward.

In this expression, $A_{x_{1}}\left(x_{0}, y_{0}, z_{0}\right)$ instead of being given by eq 30 is now given by

$$
A_{x_{1}}\left(x_{0}, y_{0}, z_{0}\right)=\mp \int_{-\frac{l_{1}}{2}}^{\frac{l_{1}}{2}} \frac{d x_{1}}{R}
$$

where $R$ is the distance from $x_{0}, y_{0}, z_{0}$ to $d x_{1}$, and the upper sign is taken when the axial current in sheet No. 1 is positive (to the right).

If the lead wire is above the solenoid $R^{2}=\left(x_{1}-x_{0}\right)^{2}+\left(a_{1}-y_{0}\right)^{2}$, where $x_{0}=x_{2} \cos \theta$ and $y_{0}=x_{2} \sin \theta$. If the lead wire is below the solenoid $R^{2}=\left(x_{1}-x_{0}\right)^{2}+\left(a_{1}+y_{0}\right)^{2}$.

In the first case, it is found that

$$
A_{x_{1}}\left(x_{0}, y_{0}, z_{0}\right)=\mp \log \frac{\sqrt{r_{1}^{2}-2 r_{1} x_{2} \cos \left(\theta-\alpha_{1}\right)+x_{2}^{2}}+r_{1} \cos \alpha_{1}-x_{2} \cos \theta}{\sqrt{r_{1}^{2}+2 r_{1} x_{2} \cos \left(\theta+\alpha_{1}\right)+x_{2}^{2}}-r_{1} \cos \alpha_{1}-x_{2} \cos \theta}
$$

By changing the sign of $\alpha_{1}$ in this, it applies for the second case where the lead wire is below the solenoid. Since $\left(\frac{x_{2}}{r_{1}}\right)^{2}$ is negligible, this reduces to

$$
A_{x_{1}}\left(x_{0}, y_{0}, z_{0}\right)=\mp 2\left\{\log \left(\frac{l_{1}+\sqrt{l_{1}^{2}+4 a_{1}^{2}}}{2 a_{1}}\right)+\frac{x_{2} \sin \theta \cos \alpha_{1}}{a_{1}}\right\}
$$

If the wire is below the solenoid the last term has the negative sign. However, this term disappears in the integration with respect to $x_{2}$, so that both positions of the lead-in wire have the same effect. On substituting this value of $A_{x}\left(x_{0}, y_{0}, z_{0}\right)$ in eq 35 , it must be remembered 
that the ambiguities in sign do not represent the same alternative. Hence

$$
m_{l_{1} f_{3}}=\mp 2 \mu\left\{\log \left(\frac{l_{1}+\sqrt{l_{1}^{2}+4 a_{1}^{2}}}{2 a_{1}}\right)+\frac{x_{2} \sin \theta \cos \alpha_{1}}{a_{1}}\right\}
$$

and

$$
m_{l_{1} s_{9}}(\mu)=\int_{-\frac{l_{2}}{2}}^{\frac{l_{3}}{2}} m_{l_{1} \rho_{3}} d x_{2}=\mp 2 \mu l_{2}, \log \left(\frac{l_{1}+\sqrt{l_{1}^{2}+4 a_{1}^{2}}}{2 a_{1}}\right)
$$

The contribution of this lead wire to the torque is $t_{l_{1} s_{2}}=\left(D_{\mu} m_{l 1 s_{2}}\right)_{\mu=0}$ or

$$
t_{l_{1} s_{2}}= \pm 2 l_{2} \log \left[\frac{l_{1}+\sqrt{l_{1}^{2}+4 a_{1}^{2}}}{2 a_{1}}\right]
$$

In this equation the upper sign applies when the axial currents on the solenoids are both positive or both negative (i. e., in the direction of increasing $x_{1}$ or $x_{2}$ of fig. 1). In this case the upper sign applies to eq $34^{\prime}$. A comparison of eq 40 and $34^{\prime}$ shows that the correction for axial components of current is compensated for by this arrangement of lead-in wires.

\section{SUMMARY}

The torque between concentric solenoids with axes at right angles is given by eq 17 , in which the integrations enable one to correct for small observed variations in diameter and spacing of the turns of wire in the two coils. The remainder of eq 17 is the torque between two current sheets, the term $S$ being computed by eq $12^{\prime}$. The fact that the coils consist of discrete turns of wire of radii $\rho_{1}$ and $\rho_{2}$ (and not of tape windings with no insulation space between them as in current sheets) is taken into account by using the "effective" or "electrical" radii $a_{1}$ and $a_{2}$ of the coils in computing the torque between the sheets. The mean diameter, $2 \bar{a}_{i}$, of a coil is the over-all diameter diminished by the diameter $2 \rho_{i}$ of the wire, and its effective diameter is $2 a_{i}$ where, by eq $9, a_{i}=\bar{a}_{i}\left[1 \pm \frac{1}{8}\left(\frac{\rho_{i}}{\bar{a}_{i}}\right)^{2}\right]$ the positive sign applying if the current is uniformly distributed over the section of the wires; the minus sign, if it is the natural distribution (inversely proportional to the distance from the axis). The same results hold if the section of the wire is not round but symmetrical with respect to its center of gravity (such as a square), provided that $\rho_{i} / 2$ then denotes the radius of gyration of the section.

The correction terms are derived on the assumption that the outer coil is relatively long, and that its magnetic field is nearly uniform over the region occupied by the inner coil. Application is made to the case where the coil is compressed more at its middle than at its ends because of the tension in the windings. This gives the correction term in eq 22. The effect of errors in centering the coils is shown to be generally negligible, although formula 17 enables one to compute these errors if necessary. 
Since a single-layer coil is necessarily helical, the axial components of current in both coils contribute a small term to the torque whose value is found by eq $34^{\prime}$. It amounts to about 3 parts in one hundred thousand. It is shown by eq 40 that this torque is practically compensated for, when the lead-in wire of the outer coil is very near to it (either above or below) and lies in the plane containing the axes of the coils.

The derivation of these formulas was undertaken, having in mind their application to a proposed absolute measurement of current in the Pellat type of current balance to be made at the National Bureau of Standards.

The formulas have also (unexpectediy) been found useful by E. A. Johnson of the Department of Terrestial Magnetism, who devised a method of absolute measurement of the earth's magnetic fiald, by uniform rotation of the secondary. Instead of measuring torque, he observed the induced electromotive force, both of which however, require the differentiation of the mutual inductance with respect to the angle, $\theta$.

Washington, January 31, 1939. 